\title{
Study on Turbulence Effects for Beam Propagation in Turbulent Atmosphere
}

\author{
Pingping Pan \\ College of physics and communication electronics, Jiangxi normal university, Nanchang, 330022
}

Received 2013

\begin{abstract}
Based on the theory for the turbulence and the Rytov method, the propagation formulas of the scintillation index and the mean square angle-of-arrival fluctuation for the beam propagation in atmospheric turbulence have been derived respectively. The propagation properties of the two turbulence effects have been investigated, and the effects of the characteristic parameters of turbulence and the beam parameters have been discussed. The results show that the variation of the two turbulence effects depends on the structure constant of the refractive index fluctuations $\mathrm{Cn} 2$, the inner scale of the turbulence 10, the waist width of source beam w0 and the wave length $\lambda$. Moreover, there are two parameters including $\mathrm{Cn} 2$ and 10 which show more significant effects in atmosphere. Consequently, a new method for determining the characteristics parameters of the turbulence by using the measurement of the scintillation index and the angle-of-arrival fluctuation has been proposed.
\end{abstract}

Keywords: Atmospheric Turbulence; Rytov Method; Scintillation Index, Mean Square Angle-of-Arrival Fluctuations; Constant of the Refractive Index Fluctuations

\section{Introduction}

The study on the propagation of laser beams through turbulence is of great importance for many practical applications, such as the remote sensing, atmospheric optical communications, and track system [1-5]. There are a few affecting factors on the beam propagation in atmosphere, such as atmosphere attenuation, atmospheric absorption, scattering, and atmospheric turbulence effect etc. The atmospheric turbulence effects include the beam spreading, the angle-of-arrival fluctuations, the scintillation index and the bit error rate, and they have more obvious influence on the performance of a communication system. [6-10].

The scintillations and the angle-of-arrival fluctuations are more significant effects of atmospheric turbulence on the beam propagation. The effect of the angle of arrival may increase the field-of-view requirements in a direct-detection optical receiver and degrade the performance a heterodyne system [11-13]. It is well known that the atmospheric turbulence affects the received intensity, resulting in the intensity fluctuations at the receiver plane. Consequently, the optical scintillation take place in atmospheric optic links [14-15]. In this paper, the propagation properties of the scintillations and the angle-of-arrival fluctuations have been investigated, the effects of the turbulent parameters and the beam parameters have been discussed, and a method for estimating the characteristics parameters of the turbulence has been proposed.

\section{Turbulence Effects for Beam Propagation in Atmosphere}

\subsection{Mean Square Angle-of-Arrival Fluctuations}

When light travels through the atmosphere, it experiences phase fluctuations due to turbulence. The mean square angle-of-arrival fluctuation is related to the phase structure function through the expression below [14],

$$
<\alpha_{a}^{2}>=\frac{D_{s}\left(\mathbf{p}_{1}=0,\left|\mathbf{p}_{2}\right|=R, L\right)}{(k R)^{2}}
$$

Here the mean square angle-of-arrival fluctuations are evaluated at a radial distance $R, L$ is the path length, i.e., the length of the atmospheric link, and $k=2 \pi / \lambda$ is the wave number, $\lambda$ is wavelength. The second phase point lies at

$$
\mathbf{p}_{2}=\left(p_{x_{2}}, p_{y_{2}}\right),\left|\mathbf{p}_{2}\right|=R, \mathbf{p}_{1}=\left(p_{x_{1}}, p_{y_{1}}\right)=(0,0)
$$

The phase structure function described as

$$
D_{s}\left(\mathbf{P}_{1}, \mathbf{P}_{2}, L\right)=\left\langle\left[S\left(\mathbf{P}_{1}, L\right)-S\left(\mathbf{P}_{2}, L\right)\right]^{2}\right\rangle
$$

where $<>$ denotes the ensemble average over the medium statistics,

$$
S(\mathbf{p}, L)=\frac{1}{2 j}[\psi(\mathbf{p}, L)-\psi *(\mathbf{p}, L)]
$$

the asterisk represents the complex conjugate. In Rytov method, $\psi$ denotes the fluctuations of the complex am- 
plitude in turbulence [4],

$$
\begin{aligned}
\psi(\mathbf{p}, L)= & \frac{k^{2}}{2 \pi E^{\mathrm{FS}}(\mathbf{p}, L)} \int_{V^{\prime}} d^{3} r^{\prime} n_{1}\left(\mathbf{p}^{\prime}, z^{\prime}\right) E^{\mathrm{FS}}\left(\mathbf{p}^{\prime}, z^{\prime}\right) \\
& \times \frac{\exp \left(i k\left|\mathbf{r}-\mathbf{r}^{\prime}\right|\right)}{\left|\mathbf{r}-\mathbf{r}^{\prime}\right|}
\end{aligned}
$$

$E^{\mathrm{FS}}(\mathbf{P}, z)$ is the field in free space and

$$
\begin{gathered}
\mathbf{r}^{\prime}=\left(\mathbf{P}^{\prime}, z^{\prime}\right)=\left(p_{x}{ }^{\prime}, p_{y}{ }^{\prime}, z^{\prime}\right), d \mathbf{r}^{\prime}=d p_{x}{ }^{\prime} d p_{y}{ }^{\prime} d z^{\prime} \\
n_{1}\left(\mathbf{p}^{\prime}, z^{\prime}\right)=n_{1}\left(p_{x}{ }^{\prime}, p_{y}{ }^{\prime}, z^{\prime}\right)
\end{gathered}
$$

expresses the random part of the refractive index, which can be written in the form of a Fourier-Stieltjes representation [5] for a statistically homogeneous and isotropic medium.

The field distribution of Gaussian beam can be written as,

$$
E\left(x_{1}^{\prime}, y_{1}^{\prime}, 0\right)=\exp \left(-\frac{x_{1}^{\prime 2}+y_{1}^{\prime 2}}{w_{0}^{2}}\right)
$$

where $w_{0}$ is the waist width and $\left(x_{1}^{\prime}, y_{1}^{\prime}\right)$ is the transversal coordinates at the $z=0$ plane. Then, by using the paraxial form of the extended Huygens-Fresnel principle, the field distribution of Gaussian beam through free space can be obtained,

$$
\begin{array}{r}
E^{F S}\left(x_{1}, y_{1}, z\right)=\frac{k w_{0}^{2}}{k w_{0}^{2}+2 i z} \exp \left[i k z+\frac{i k}{2 z}\left(x_{1}^{2}+y_{1}^{2}\right)\right. \\
\left.-\frac{k^{2} w_{0}^{2}\left(x_{1}^{2}+y_{1}^{2}\right)}{4 z^{2}-2 i k z w_{0}^{2}}\right]
\end{array}
$$

Assume that the turbulent atmosphere is statistically homogenous and isotropic, the source and the medium statistics are independent. The mean square angle-ofarrival fluctuation of Gaussian beam in turbulence can be derived,

$$
\begin{array}{r}
<\alpha_{a}^{2}>=0.1628 C_{n}^{2} L \kappa_{0}^{1 / 3}\left(-14.4+5.5663\left(\frac{\kappa_{m}}{\kappa_{0}}\right)^{1 / 3}\right. \\
\times \int_{0}^{1} d t \operatorname{Re}\left\{\left[\frac{1}{1+\kappa_{m}^{2} L^{2}(1-t)^{2} \xi_{1}}\right]^{1 / 6} \gamma^{2}\right. \\
\left.\left.+\left[\frac{k}{k+j \gamma \kappa_{m}^{2} L(1-t)}\right]^{1 / 6} \gamma^{2}\right\}\right)
\end{array}
$$

Re means the real part, $C_{n}{ }^{2}$ is the structure constant of the refractive index fluctuations of the turbulence. $\kappa_{m}$ $=5.92 / l_{0}, l_{0}$ is the inner scale of turbulence; $\kappa_{0}=1 / L_{0}, L_{0}$ is the outer scale of turbulence. And

$$
\xi_{1}=\frac{1}{k^{2} w_{0}^{2}+\left(L / w_{0}\right)^{2}}, \gamma=\frac{k w_{0}^{2}+j L t}{k w_{0}^{2}+j L}
$$

\subsection{Scintillation Index}

The scintillation index in atmospheric turbulence at the receiver plane is given by [8-10]

$$
\begin{gathered}
\sigma_{I}^{2}=4 B_{\chi}\left(\mathbf{p}_{1}, \mathbf{p}_{2}, z=L\right) \\
B_{\chi}\left(\mathbf{p}_{1}, \mathbf{p}_{2}, z=L\right)=<\chi\left(\mathbf{p}_{1}, L\right) \chi\left(\mathbf{p}_{2}, L\right)>
\end{gathered}
$$

is the correlation function of the log-amplitude fluctuations at the receiver plane $z=L$,

$$
\chi(\mathbf{p}, L)=0.5[\psi(\mathbf{p}, L)+\psi *(\mathbf{p}, L)]
$$

and $\mathrm{B} \chi$ can be written as

$$
B_{\chi}=\pi \int_{0}^{z} d \eta \int_{-\infty}^{\infty} \int_{-\infty}^{\infty}\left[\operatorname{Re}(\phi \phi)+\operatorname{Re}\left(\phi^{*} \phi\right)\right] \Phi_{n}(\kappa) d \kappa_{x} d \kappa_{y}
$$

$\Phi_{n}(\kappa)$ denotes the spatial power spectra of the refractive index fluctuations of the turbulent atmosphere, $\Phi_{n}(\kappa)=0$ in free space, and $\kappa=\left(\kappa_{\mathrm{x}}{ }^{2}+\kappa_{\mathrm{y}}{ }^{2}\right)^{1 / 2}$.

$$
\begin{aligned}
\phi= & \frac{-i k}{E^{F S}(0, z)} \exp (i k z) \frac{k w_{0}^{2}}{2 i z+k w_{0}^{2}} \\
& \times \exp \left[\frac{(z-L t)\left(2 L t-i k w_{0}^{2}\right) \kappa^{2}}{2 k\left(2 i z+k w_{0}^{2}\right)}\right]
\end{aligned}
$$

Letting $x=0$ and $y=0$ in $E^{F S}(x, y, z)$, the on-axis field distribution $E^{F S}(0, z)$ can be obtained. Tatarskii power spectra is represented as

$$
\Phi_{n}(\kappa)=0.033 C_{n}^{2} \kappa^{-11 / 3} \exp \left(-\frac{\kappa^{2}}{\kappa_{m}^{2}}\right)
$$

After some tedious integral calculation, the scintillation index of Gaussian beam in turbulence can be derived,

$$
\begin{aligned}
\sigma_{I}^{2}= & 0.8816 \pi^{2} C_{n}^{2} k^{2} L \operatorname{Re} \times \int_{0}^{1}\left\{\left[\frac{2 w_{0} L^{2}(t-1)^{2}}{k\left(1+4 L^{2} w_{0}^{2}\right)}+\frac{1}{k_{m}^{2}}\right]\right. \\
& \left.-\left[\frac{L(t-1)\left(1+2 i L t w_{0}\right)}{i k\left(1+2 i L w_{0}\right)}+\frac{1}{k_{m}^{2}}\right]\right\}^{5 / 6} d t
\end{aligned}
$$

\section{Numerical Examples and Analysis}

\subsection{Angle-of-Arrival Fluctuations}

In this section, the variation of the angle-of-arrival fluctuations have been studied, and the effects of the turbulent parameters and the beam parameters have been discussed. Eq. (10) is numerically evaluated and used in plotting the root mean square of the angle-of-arrival (RMS-AOA) fluctuations. Figure 1 gives the variation of the angle-of-arrival fluctuations, and the calculation parameters are $C_{n}^{2}=10^{-14} \mathrm{~m}^{-2 / 3}, w_{0}=30 \mathrm{~mm}, \lambda=1.55 \mu \mathrm{m}, L$ $=3 \mathrm{~km}, l_{0}=10 \mathrm{~mm}, L_{0}=25 \mathrm{~m}$.

The wave front tilt of optical wave propagation through atmospheric turbulence gives rise to an angle-of-arrival fluctuation. Figure 1(a) shows the effects of constant of the refractive index fluctuations, $C=C_{n}^{2} / 10^{-15} \mathrm{~m}^{-2 / 3}$. The RMS-AOA is zero in the initial plane, and it increases 
versus the propagation distance $L$. The RMS-AOA increases when beams propagate through stronger turbulence, which can be revealed from Eq. (10) straightway. In addition, the effect of the turbulence is more obvious for larger $L$.

The effects of the inner scale of the turbulence $l_{0}$ and the outer scale of the turbulence $L_{0}$ are displayed in Figure 1(b). The change range of the inner scale $l_{0}$ in turbulence has been adopted between $1 \mathrm{~mm}$ and $20 \mathrm{~mm}$ usually, so the variation of RMS-AOA under the change range of $l_{0}$ is represented. Examining Figure 1(b), the RMS-AOA decreases for the larger $l_{0}$ and smaller $L_{0}$.
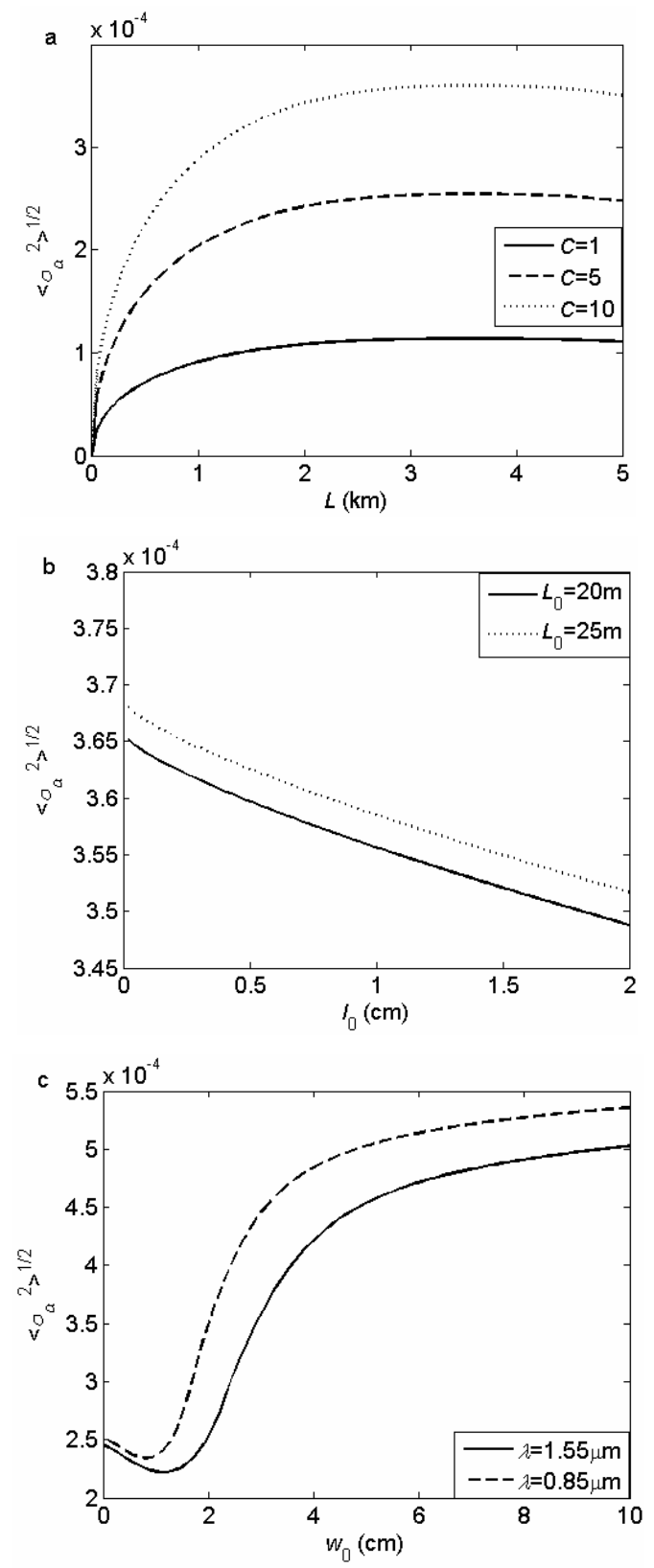

Figure 1. Variation of the angle-of-arrival fluctuations in turbulence.
Figure 1(c) provides the effects of the beam parameters including the waist width $w_{0}$ and wavelength $\lambda$. The RMS-AOA decreases first and increases then, and there exits a minimum during the variation of $w_{0}$. In our graphs, two infrared wavelengths are employed, representing the most commonly utilized wavelengths in current space links. From Figure 1(c), the RMS-AOA of the beam with $\lambda=0.85 \mu \mathrm{m}$ is larger than $\lambda=1.55 \mu \mathrm{m}$. Moreover, the waist width where the value of minimum for the RMS-AOA happens is smaller. If the waist width is very big, the RMS-AOA of the beam will increase. On the other hand, if $w_{0}$ is very small, it is not easy to obtain larger power in the initial plane, so the propagation distance is limited in practice. Therefore, the appropriate waist width and wavelength should be selected.

\subsection{Scintillations}

Scintillations reflect the intensity fluctuations at the receiver plane, and the variation of the scintillation index in turbulence is provided in Figure 2. The calculation parameters $C_{n}^{2}=10^{-14} \mathrm{~m}^{-2 / 3}, w_{0}=30 \mathrm{~mm}, \lambda=1.55 \mu \mathrm{m}, L$ $=3 \mathrm{~km}, l_{0}=10 \mathrm{~mm}$ are taken. In Figure 2(a), the scintillation index rises against propagation distance and through stronger turbulence. Furthermore the effect of the turbulence on the scintillation index is more obvious when the propagation distance increases. The variation of scintillation index given in this paper is consistent with the results of
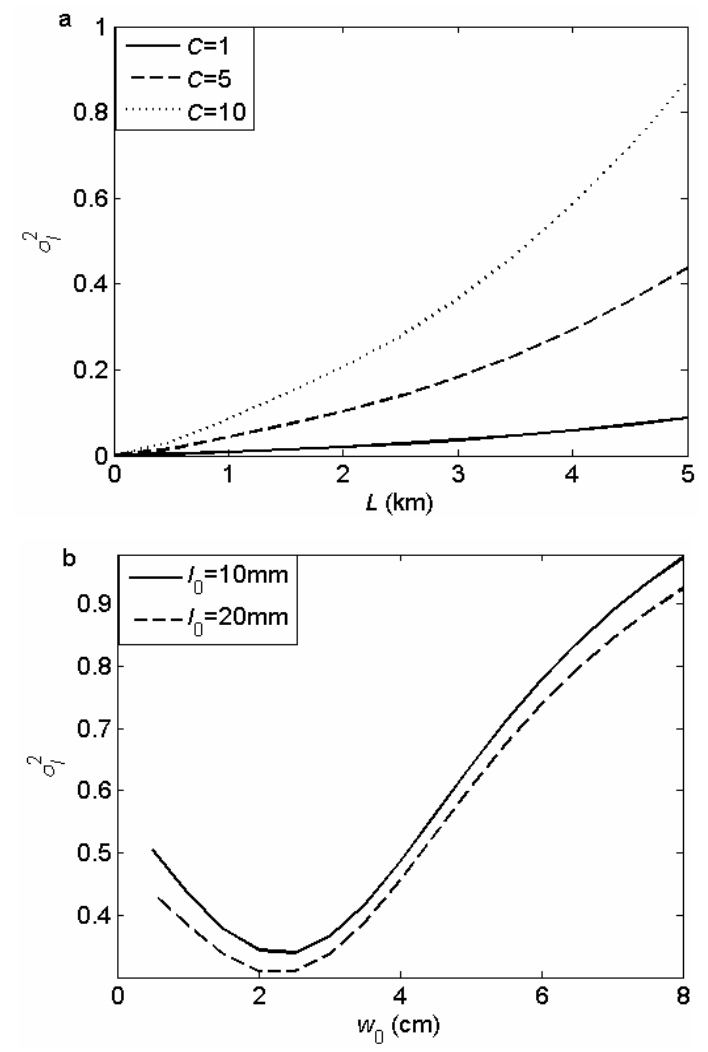

Figure 2. Variation of the scintillations in turbulence. 
related literature [13]. As observed from Figure 2(b), there exits a minimum for the scintillation index during the variation of $w_{0}$, and both the scintillation index and the corresponding minimum are larger for the smaller $l_{0}$.

In a word, the effect of the turbulence on beam propagating in turbulence is very obvious, so the beam quality may be affected, and especially the effect of the constant of the refractive index fluctuations is not neglected. Consequently, it is significative to estimate the structure constant of the turbulence.

\subsection{Estimation of the Structure Constant of the Turbulence}

The relations of the turbulence effects and turbulent parameters are illustrated in Figure $\mathbf{3}$ and Figure $\mathbf{4}$ respectively, which implies a method for the estimation of the structure constant of the turbulence. We noted that in our solution, the change range of the inner scale $l_{0}$ is adopted between $1 \mathrm{~mm}$ and $20 \mathrm{~mm}$, whereas the range of the structure constant spans several magnitudes. Firstly, if the RMS-AOA is measured by experiments, by using the relation of the RMS-AOA and turbulent parameters (see Figure 3), the structure constant of the turbulence can be estimated roughly. Secondly, if the scintillation index is measured by experiments, by using the relation of the scintillation index and turbulent parameters (see Figure 4), $C_{n}{ }^{2}$ can be estimated once again. Applying the two change ranges, we can estimate the range of the structure constant of the turbulence more accurately.

\section{Conclusions}

In this paper, the propagation formulas of the angle-ofarrival fluctuation and the scintillation index for the beam propagation in atmospheric turbulence have been obtained respectively, indicating that there are similarities in the derivation of the RMS-AOA and the scintillation

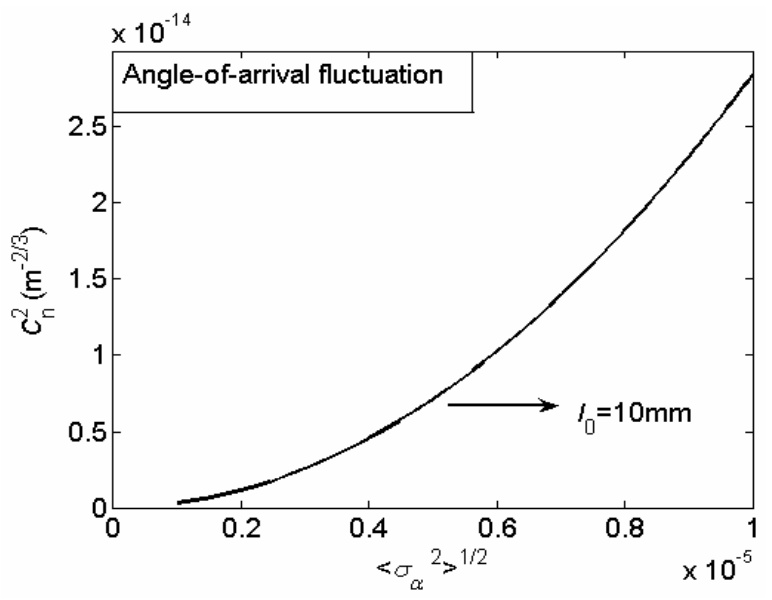

Figure 3. Relation of the RMS-AOA and turbulent parame- ters.

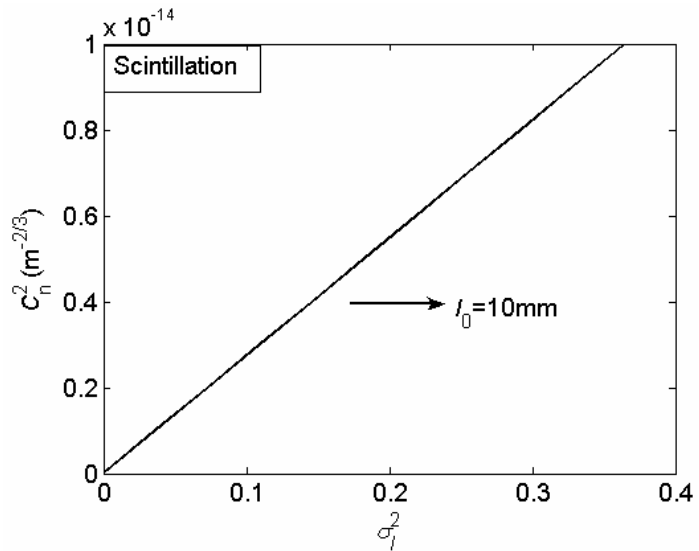

Figure 4. Relation of the scintillation index and turbulent parameters.

index. The effects of the turbulent parameters and the beam parameters have been analyzed quantitatively. The angle-of-arrival fluctuation and the scintillation index rise with the increasing propagation distance and the structure constant of the turbulence, and the decreasing inner scale, moreover there exits a minimum for them during the variation of waist width. Finally, the range of the structure constant of the turbulence can be estimated from the RMS-AOA and the scintillation index measured by experiments.

\section{REFERENCES}

[1] Y. Cai, H. T. Eyyuboğlu and Y. Baykal, "Propagation properties of anomalous hollow beams in a turbulent atmosphere," Optics Communications, Vol. 281, No. 21, 2008, pp. 5291-5297

doi:10.1016/j.optcom.2008.07.080

[2] Y. Dan, B. Zhang and P. pan, "Propagation of Partially Coherent Flat-Topped Beams Through A Turbulent Atmosphere," Journal of Optical Society of America A, Vol. 25, No.9, 2008, pp. 2223-2231. doi:10.1364/JOSAA.25.002223

[3] P. Pan, Y. Dan, B. Zhang, N. Qiao and Y. Lei, "Propagation Properties of Flat-Topped Beams in A Turbulent Atmosphere," Journal of Modern Optics, Vol. 56, No. 12, 2009,pp.1375-1382.doi:10.1080/09500340903145049

[4] A. Ishimaru, "Fluctuations in the Parameters of Spherical Waves Propagating in A Turbulent Atmosphere," Radio Science, Vol. 4, 1969, pp. 209-305.

[5] A. Ishimaru, Wave Propagation and Scattering in Random Media, New York San Francisco, London, 1978, pp. 93-101.

[6] G. Gbur and E. Wolf, "Spreading of Partially Coherent Beams in Random Media," Journal of the Optical Society of America A, Vol. 19, No.8, 2002, pp. 1592-1598. doi:10.1364/JOSAA.19.001592

[7] X. Ji, X. Li, S. Chen, E. Zhang and B. Lü, "Spatial Cor- 
relation Properties of Gaussian-Schell Model Beams Propagating through Atmospheric Turbulence," Journal of Modern Optics, Vol. 55, 2008, pp. 877-891. doi: 10.1080/09500340701504314

[8] H.T. Eyyuboğlu, Y. Baykal and X. Ji, "Scintillations of Laguerre Gaussian beams," Applied Physics B, Vol. 98, No. 4, 2009, pp. 857-863. doi:10.1007/s00340-009-3702-X

[9] Y. Cai, Y. Chen, H. T. Eyyuboğlu and Y. Baykal, "Scintillation Index of Elliptical Gaussian Beam in Turbulent Atmosphere," Optics Letters, Vol. 32, 2007, pp. 2405-2407. doi:10.1364/OL.32.002405

[10] Y. Chen, Y. Cai, H. T. Eyyuboğlu and Y. Baykal, "Scintillation Properties of Dark Hollow Beams in A Weak Turbulent Atmosphere," Applied Physics B, Vol. 90, 2008, pp. 87-92. doi:10.1007/s00340-007-2825-1

[11] Y. Baykal and H. T. Eyyuboğlu, "Scintillation Index of Flat-Topped Gaussian Beams," Applied Optics, Vol.45,
No.16, 2006, pp. 3793-3797. doi:10.1364/AO.45.003793

[12] P. Pan, B. Zhang, N. Qiao and Y. Dan, "Characteristics of Scintillations and Bit Error Rate of Partially Coherent Rectangular Array Beams in Turbulence," Optics Communications, Vol.284, 2011, pp. 1019-1025. doi:10.1016/j.optcom.2010.10.053

[13] Y. Chen, Y. Cai, H. T. Eyyuboğlu, Y. Baykal, "Scintillation Properties of Dark Hollow Beams in A Weak Turbulent Atmosphere," Applied Physics B, Vol. 90, 2008, pp. 87-92. doi:10.1007/s00340-007-2825-1

[14] Y. Zhang and C. Tao, "Angle-of-arrival of Gaussian Schell Beam Propagation in Atmospheric Turbulence," Acta Photonica Sinica, Vol. 34, 2005, pp. 424-427.

[15] J. Ma, C. Gao and L. Tan, "Angle-of-arrival Fluctuations in Moderate to Strong Turbulence," Chinese Physics, Vol. 16, 2007, pp. 1327-1333. doi:10.1088/1009-1963/16/5/027 